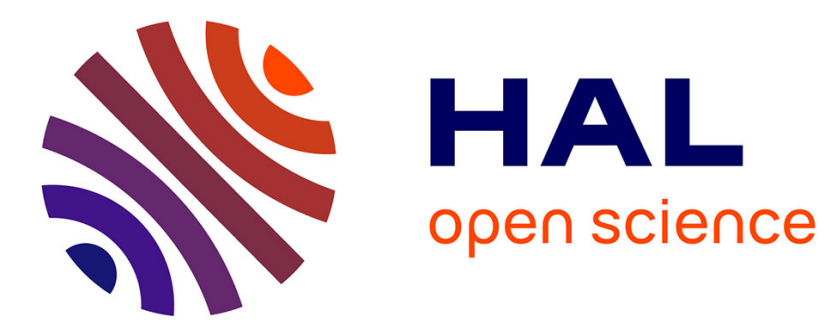

\title{
Observation of a new memory effect in a modulated structure
}

\author{
J.P. Jamet, P. Lederer
}

\section{To cite this version:}

J.P. Jamet, P. Lederer. Observation of a new memory effect in a modulated structure. Journal de Physique Lettres, 1983, 44 (7), pp.257-264. 10.1051/jphyslet:01983004407025700 . jpa-00232190

\section{HAL Id: jpa-00232190 https://hal.science/jpa-00232190}

Submitted on 1 Jan 1983

HAL is a multi-disciplinary open access archive for the deposit and dissemination of scientific research documents, whether they are published or not. The documents may come from teaching and research institutions in France or abroad, or from public or private research centers.
L'archive ouverte pluridisciplinaire HAL, est destinée au dépôt et à la diffusion de documents scientifiques de niveau recherche, publiés ou non, émanant des établissements d'enseignement et de recherche français ou étrangers, des laboratoires publics ou privés. 
Classification

Physics Abstracts

$61.70-64.70 \mathrm{~K}-66.30 \mathrm{~J}$

\title{
Observation of a new memory effect in a modulated structure (*)
}

\author{
J. P. Jamet and P. Lederer \\ Laboratoire de Physique des Solides (**), Université Paris-Sud, Bâtiment 510, 91405 Orsay, France
}

(Reçu le 23 septembre 1982, révisé les 15 décembre 1982 et 20 janvier 1983, accepté le 10 février 1983)

\begin{abstract}
Résumé. - Nous décrivons la première observation d'un nouvel effet de mémoire dans une structure modulée (thiourée deutériée). Nous présentons une explication qualitative de cet effet basée sur l'interaction de défauts ou d'impuretés mobiles avec le paramètre d'ordre de la modulation. Nous montrons que cet effet peut être utilisé pour obtenir les lignes d'égal vecteur d'onde de modulation dans le plan $E, T$ du diagramme de phase par des méthodes thermodynamiques. Nous suggérons que les systèmes à $\mathrm{ODC}$ comme $\mathrm{NbSe}_{3}$ présentent eux aussi une modulation périodique de concentration de défauts, ce qui rendrait compte des effets non linéaires en champ électrique. (ODC : Onde de Densité de Charge.)
\end{abstract}

\begin{abstract}
We report the first observation of a new memory effect in a modulated structure (deuterated thiourea). We present a qualitative explanation of this effect in terms of mobile defects, or impurities, interacting with the modulation order parameter. We show that this effect can be used to obtain the lines of constant modulation wavevector in the $E, T$ plane of the phase diagram, by thermodynamic methods. We suggest that a similar periodic modulation of defect concentration occurs in $\mathrm{CDW}$ systems, like $\mathrm{NbSe}_{3}$, and accounts for the non linearity induced by the electric field. (CDW : Charge Density Wave.)
\end{abstract}

Thermal hysteresis effects are present in ferroelectrics and modulated structures and are known to exist in dipole density wave systems such as $\mathrm{SC}\left(\mathrm{ND}_{2}\right)_{2}$ [1], $\mathrm{Rb}_{2} \mathrm{ZnCl}_{4}$ [2], $\mathrm{TMAZnCl}_{4}$ [3], $\mathrm{BaNaNb}_{5} \mathrm{O}_{15}$ [4] or charge density wave systems such as TTF-TCNQ [5]. They are observed by using neutron diffraction, specific heat, dielectric susceptibility or linear birefringence experiments. The effect of impurities by doping has been investigated for example in $\mathrm{Rb}_{2-x} \mathrm{~K}_{x} \mathrm{ZnCl}_{4}$ [6] but there is, to our knowledge, no experimental study concerning the effect of dislocations or tunnelling impurities on the properties of a modulated structure. In some systems [4], the rather large thermal hysteresis which is observed on the $q$-wavevector evolution of the modulation plays in favour of the influence of impurities or defects on the modulated structure. The pinning of the incommensurate modulation by the defects has been investigated from a theoretical point of view in the case of the charge density waves [7].

(*) Le contenu de cet article a été présenté en partie au Colloque du C.N.R.S. : Parois et Domaines (RCP 608) Aussois, janvier 1982.

$\left({ }^{* *}\right)$ Associé au C.N.R.S. 
We present here an effect observed in the modulated phase of thiourea which we call a memory effect and which can presumably be attributed to an ordering of defects or impurities or other mobile entities by the modulation order parameter; we believe that this effect which, to our knowledge, has never been observed before is tightly related to the existence of hysteresis in the following sense : if the effect is extrinsic, then hysteresis is most likely of extrinsic origin (even though different defects may explain one or other); if the effect is intrinsic, then hysteresis is probably also intrinsic.

\section{Linear birefringence and dielectric susceptibility experiments.}

The susceptibility measurements have been performed by using a Sawyer-Tower like circuit. The reference sinusoidal signal at $1.5 \mathrm{kHz}$ is given by a high stability low distortion generator and the detection uses a lock-in amplifier coupled to a X-Y recorder and a digital voltmeter; no significant difference has been found on the susceptibility between $10 \mathrm{~Hz}$ and $600 \mathrm{kHz}$ which means that $\varepsilon=\varepsilon_{0}$. For the measured sample (thickness : $0.62 \mathrm{~mm}$, area : $6 \mathrm{~mm}^{2}$ ) the Noise to Signal Ratio (N.S.R.) was $2 \times 10^{-4}$, while the kink amplitude, in the case of figure 1 , represents about 10 times the noise amplitude (furthermore the absolute precision on the susceptibility measurement is certainly not better than $10^{-2}$ ). On the other hand, the birefringence measurements on the same sample used an acousto-optical modulator to improve the signal to noise ratio [8]; the light source is a stabilized HeNe Laser $(\lambda=6328 \AA)$; the optical phase shift is $\simeq 15 \mathrm{radians} / \mathrm{mm}$ [9] in the modulated phase (i.e. between $190 \mathrm{~K}$ and $216 \mathrm{~K}$ ) and the noise amplitude is $10^{-3}$ radian $/ \mathrm{mm}$; as an example the kink amplitude is about $5 \times 10^{-3} \mathrm{radian} / \mathrm{mm}$ if $T_{0}=194 \mathrm{~K}$, but it is about $10^{-3} \mathrm{radian} / \mathrm{mm}$ at $202 \mathrm{~K}$. We report here the most accurate results obtained by susceptibility. Birefringence results will be reported elsewhere.

In the modulated phase of thiourea $(190 \mathrm{~K}<T<216 \mathrm{~K}$, in zero d.c. field and at atmospheric pressure) the dielectric susceptibility and the birefringence exhibit a large thermal hysteresis :

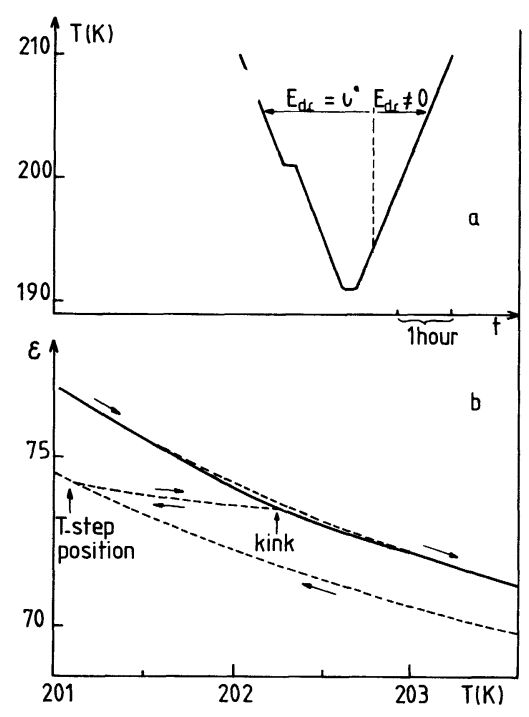

Fig. 1. - a) Typical temperature evolution as a function of time used to get the memory effect. The step located around $201 \mathrm{~K}$ during the cooling phase will generate a kink during the heating phase at a larger temperature $T \sim 202 \mathrm{~K}$ (due to the hysteresis effect).

b) Experimental susceptibility curves for a cleaved sample with dimensions $2 \times 3 \mathrm{~mm}^{2}$, thickness $0.62 \mathrm{~mm}$. The dotted lines represent the curve obtained during a continuous run and the solid line the curve obtained by using the procedure described above. $E_{\text {d.c. }}=0, E_{\text {a.c. }}=10 \mathrm{mV} / \mathrm{cm}$ at $1.5 \mathrm{kHz}$. 


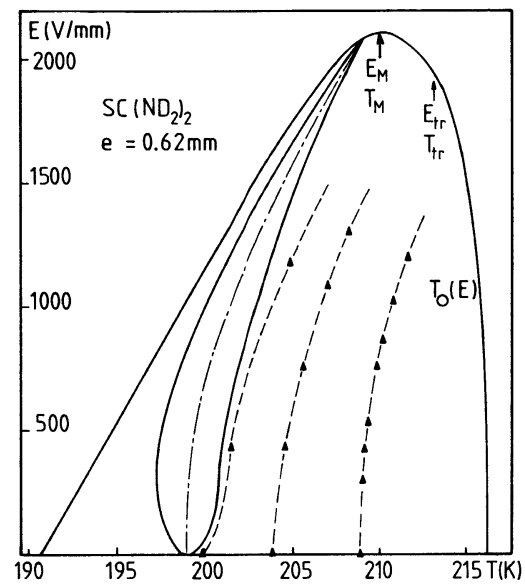

Fig. 2. $-E, T$ phase diagram of $\operatorname{SC}\left(\mathrm{ND}_{2}\right)_{2}$; the solid lines are from [10]. The flame-shaped curve is the envelop of the commensurate phase with $q=b^{*} / 8$ and the triangles are obtained by using the memory effect ; the dotted lines are just a help to the eyes, they join the points obtained by using the same temperature step during the cooling phase and applying various field values during the heating phase which result in various kink positions. The theoretical analysis given in [12] for the $q=b^{*} / 8$ phase assumed, in the absence of Umklapp term, a theoretical line (dashed dotted line) which is also the middle line of the $q=b^{*} / 8$ commensurate phase; the excellent agreement of the theoretical and experimental curvatures is an excellent check of the theory given in [12].

it is null within experimental accuracy at and above the second order phase transition temperature $\left(T_{0} \simeq 216 \mathrm{~K}\right)$ separating the paraelectric and modulated phases and increases monotonically below $T_{0}$ down to the commensurate phase transition $\left(q=b^{*} / 9\right)$ at $T_{9} \simeq 192 \mathrm{~K}$. At $190 \mathrm{~K}$, a first order transition to the ferroelectric phase $(q=0)$ occurs and below this temperature the hysteresis effect is associated to the presence of ferroelectric domain walls. If a non-zero d.c. field $E$ is applied parallel to the ferroelectric axis $" a$ », the situation is not drastically modified apart from the onset of the field-induced commensurate phase with $q=b^{*} / 8[10]$. Inside the domains of stability of the commensurate phases $\left(q=b^{*} / 8\right.$ and $\left.q=b^{*} / 9\right)$, the hysteresis effect as measured by birefringence or susceptibility experiments is different from zero.

\section{Thermal cycle and memory effect.}

Standard measurements of the susceptibility in the modulated phase (i.e. between $190 \mathrm{~K}$ and $216 \mathrm{~K}$ ) involve usually a slow $(<3 \mathrm{mK} / \mathrm{s})$ and continuous heating rate from the low temperature to the high temperature phase boundary and vice versa. As is well known, the resulting curves exhibit a thermal hysteresis. We have obtained an interesting effect when the heating (or cooling) procedure is slightly less simple, as shown on figure $1 a$. Starting first to cool in zero external field from above $216 \mathrm{~K}$ (with a constant cooling rate $|\mathrm{d} T / \mathrm{d} t|=3 \mathrm{mK} / \mathrm{s}$ ) one allows the temperature to stabilize at an arbitrary value, say $T_{0}=201 \mathrm{~K}$ during a time interval $\Delta t_{0} \sim 600 \mathrm{~s}$, then to decrease again; before going through the first order transition separating the modulated and ferroelectric phases, the temperature is again stabilized at $T_{1}=191 \mathrm{~K}$ (for a time interval which experimentally has not exceeded two days) and then increased $(\mathrm{d} T / \mathrm{d} t=3 \mathrm{mK} / \mathrm{s}):$ one then observes a small negative kink on the susceptibility or birefringence curve at a temperature slightly above $T_{0}=201 \mathrm{~K}$. The amplitude of this kink is rather small $\Delta \chi / \chi \simeq 10^{-3}$ but it is clearly visible on figure $1 b$, in a susceptibility experiment.

One may now use the same cooling procedure as above but during the heating phase, a d.c. field is applied to the sample along the « $a$ » ferroelectric axis (at a temperature such that the sam- 
ple does not become ferroelectric) then a kink is still observed but at a higher temperature than with $E_{\text {d.c. }}=0$. The loci of these kinks are displayed on figure 2 .

\section{Remarks.}

a) The experimental procedure used in $(1 a)$ can be modified for several parameters such as $T_{0}, T_{1}$, or the cooling and heating rates; the kink amplitude is smaller when $T_{0}$ is increased and it is null at and above the modulated paraelectric phase transition. If $T_{0}$ is inside the domain of stability of a commensurate phase $\left(q=b^{*} / 8\right.$, with $E_{\text {d.c. }} \neq 0$ for example) then the domain of stability of this phase appears to be slightly increased. If during the cooling process, the temperature is allowed to stabilize several times, then several kinks will be " written ". At last the kink amplitude seems to be sample dependent and consequently it is most probably related to the density of impurities or defects present inside the system; the dependence of the kink amplitude with the duration of the temperature step at $T_{0}$, has not yet been investigated quantitatively; all we can say, at present time, is that a few minutes seem to be sufficient to somehow " saturate " the memory effect.

b) The memory effect has been observed for several heating or cooling procedures, which are not reported here. A susceptibility kink not shown on figure $1 b$ is also observed upon cooling again at the locus called « $T$-step position ".

c) We do not believe that the observation of the memory effect could be an artefact due to trivial causes such as : space charge (it is observed on short-circuited samples in zero d.c. field) or electrical contact instability (the samples are gold-plated under vacuum).

\section{Interpretation.}

How can we interpret such a phenomenon ? When during the cooling process, the temperature is allowed to stabilize at $T_{0}$ (at time $t_{0}$ ), the modulation wavevector stops decreasing, so that the extrinsic defects (dislocations for example) or impurities, with relatively high mobility can relax in the periodic field of the modulated dipolar field. This can be understood on the basis of a simple steric condition : in thiourea, the modulation is a modulation of the orientation of electric dipoles (which are planar molecules) with the $b$-axis [11]; impurities (for example interstitial molecules) thus experience a modulated interaction with the lattice :

$$
V_{i}(r)=V_{0} P^{2}(r) \cdot \delta_{r, r_{i}}
$$

where $P(r)$ is the local uniaxial polarization, $\delta_{r, r_{i}}$ is the Kronecker's symbol and $r_{i}$ is the impurity position; we have developed $V_{i}(r)$ to lowest order in $P(r)$. (Thus we do not treat properly the low temperature case). $V_{0}$ is taken here to be equal for all impurities, for simplicity. (In the CDW case, it is probably more appropriate to assume $V_{i}(r) \sim \rho(r)$, where $\rho(r)$ is the charge density. In other systems, one may have more complicated impurity-modulation interactions potentials). As a result of $V_{i}(r)$, at thermal equilibrium, the impurity concentration is also periodic if the impurity mobility is not zero, with the reciprocal modulation periodicity $\left(2 q_{0}\right)^{-1}$. When the modulation is sinusoidal :

$$
C(r)=C_{0}\left[1+b_{0} \sin 2 q_{0} \cdot r\right]
$$

where $b_{0}<1 ; b_{0}$ depends on the nature of the impurities, defects, ... which can interact with the modulation potential ; also it is reasonable to assume that $b_{0}$ goes to zero with the amplitude of the modulation, i.e., as a first approximation : $b_{0} \sim\left|P_{q_{0}}\right|^{2}$ where $P_{q_{0}}$ is the Fourier transform of $P(r)$. Notice that $C(r)$ is the average concentration in the plane perpendicular to the modulation axis at point $" r \|$; in a 3 d-system, the concentration can be modulated as in the formula above even it is very small. 
When the modulation is strongly anharmonic (soliton limit), as is the case near the commensurate-incommensurate transition at $q=b^{*} / 9$, the impurity concentration profile should also exhibit a large number of harmonics and a periodic wall structure.

Thus the impurity gas can exhibit a periodic modulation with wavevector $q_{0}$ if a sufficient number of impurities has a relaxation time $\tau$ in the modulation periodic field, such that

$$
\tau<\Delta t_{0}
$$

where $\Delta t_{0}=600 \mathrm{~s}$ in the experiment described above.

a) When the temperature $T$ continues to decrease below $T_{0}=201 \mathrm{~K}$, the modulation wavevector $q(T)$ varies with $T$, i.e., with time. As a result the periodic impurity pattern is submitted to a time dependent potential. The sign of the potential-gradient, i.e. the force, alternates in time at a given impurity site, while its intensity increases with decreasing temperature due to the order parameter monotonous increase ; so if the rate of change of the temperature is sufficient, impurities do not follow the time dependent modulation, and the periodic concentration pattern keeps the initial periodicity. When the temperature becomes stationary again at $T_{1}$ at time $t_{1}$, the impurities experience a periodic potential with a different periodicity corresponding to the wavevector $q_{1}$, usually incommensurate with $q_{0}$.

At relatively short times after $t_{1}$, i.e. $t_{1}<t \lesssim t_{1}+\tau$, when the temperature is stabilized at $T=T_{1}$, the concentration pattern gets the form :

$$
C(r)=C\left(1+b_{0}^{\prime} \sin 2 q_{0} \cdot r\right)\left(1+b_{1} \sin 2 q_{1} \cdot r\right),
$$

where $b_{1}$ is different from $b_{0}$ because the order parameter varies with temperature, and where $b_{0}^{\prime}<b_{0}$. This pattern is out of equilibrium as long as $b_{0}^{\prime} \neq 0$, but we expect it to have a long lifetime because the time $\tau_{r}$ necessary to thermalize the impurities (i.e. to erase the impurity modulation with wavevector $2 q_{0}$ ) involves tunnelling of defects or impurities through the potential barriers due to the periodic modulation, so that $\tau_{r}$ is much larger than $\tau\left(\tau_{r} \gg \tau\right)$. Clearly $\tau_{r}$ increases with decreasing temperature because the height of the potential barriers increases. Therefore at time $t$ such that

$$
t_{1}+\tau<t<\tau_{r}
$$

the system exhibits a modulated concentration of impurities with fundamental Fourier components of wavevector $2 q_{0}$ and $2 q_{1}$ (and, possibly their harmonics if the dipolar modulation is not simply sinusoidal). (Note that all we can say about $\tau_{r}$ is that in our sample $\tau_{r}>48 \mathrm{~h}$ ).

b) When the temperature is increasing from $T_{1}$ (after a time shorter than $\tau_{r}$ ), there will be an extra free energy density be an extra free energy density $\delta F$, when the wavevector $q(T)$ will go through $q_{0}$. Indeed :

$$
\begin{aligned}
\delta F & =\sum_{i} V_{i}(r)=V_{0} \int_{0} b_{0}^{\prime} \sin 2 q_{0} r P^{2}(r) \mathrm{d} r \equiv F_{\mathrm{imp}} \\
& =V_{0} C_{0} b_{0}^{\prime}\left|P_{q}\right|^{2} \delta_{q, \mp q_{0}} .
\end{aligned}
$$

Note that the general expression for $\delta F$ is proportional to $\sin 2 \Phi$ where $\Phi$ ' is the phase difference between the modulation and the periodic impurity pattern. $\Phi$ is chosen so that $V_{0} b_{0}^{\prime}<0$.

(There exists also a term $b_{0}^{\prime} C_{0} P_{0} P_{q} \delta_{q, \pm 2 q_{0}}$ which we neglect here, as discussed in Appendix I.) Because of this extra term, the amplitude of the modulation experiences a small variation when $q(T)=q_{0}$ :

$$
\delta P_{q}^{2} \sim C_{0} b_{0}^{\prime} V_{0} \delta_{q(T), q_{0}}
$$


This change is reflected in the uniform susceptibility. We find (see A I) :

with

$$
\delta \chi(q=0)=V_{0} \frac{2\left(\chi_{0}\right)^{2}}{T} C_{0} b_{0}^{\prime} \delta_{q(T), q_{0}}
$$

$$
\chi_{q=0} \equiv \chi_{0}=T\left[T_{0}-T+\gamma \cdot q^{4}(T)\right]^{-1} .
$$

$\gamma$ is a phenomenological coefficient defined in A $\mathbf{I}$.

(Note that $\frac{\delta P_{q}^{2}}{P_{q}^{2}}=\frac{1}{2} \frac{\delta \chi}{\chi}$ in zero field, see appendix.)

Thus the uniform susceptibility exhibits a (negative) kink at the temperature $T_{0}(E)$ defined by $q\left[T_{0}(E)\right]=q_{0}$. Therefore, if we "write " the modulation wavevector $q_{0}$ in the system in zero field, we can determine the lines where the modulation wavevector $q_{0}$ stays constant in the $(E, T)$ plane. The preliminary results of our measurements are shown on figure 2 for three values of $q_{0}$. The results are in a very good agreement with neutron diffraction results at low d.c. field values [16] and at high field values [17].

c) When the system is heated at or above the modulated paraelectric transition $(\sim 216 \mathrm{~K}$ in zero d.c. field), the periodic field due to the modulation vanishes, so that the periodic impurity concentration relaxes to a uniform one, in a time $\Delta t \simeq \tau$. Thus if one cools the system down from high temperature, one expects no memory effect, as observed.

\section{Conclusion.}

We have described a new memory effect observed in the modulated phase of thiourea and shown that it allows to determine iso- $q$ lines in the $E-T$ plane of the phase diagram by susceptibility measurements. We have suggested that this effect can be understood if, in the crystal, there exist defects or impurities with a sufficiently broad spectrum of relaxation times, and which interact with the dipolar modulation. Likewise, iso- $q$ lines may be determined using the same effect by birefringence measurements.

Notice that the theory outlined here is a weak coupling one, in the sense that the modulationdefect coupling does not change the equilibrium value of the modulation wavevector. Situations where a strong coupling occurs should lead to a quite different behaviour.

A number of unanswered questions must still be clarified : what are the nature and the relevant relaxation times of the impurities or defects which allow to observe the memory effect ? How does the intensity of the memory effect depend on the cooling or heating rates or on the time intervals between temperature stabilizations? More fundamentally, we expect the memory effect to shed some light on the nature of the global hysteresis in thiourea : is this hysteresis intrinsic ? If it is, is it due to the Peierls potential felt by discommensuration walls [12], or is it due to the intrinsic defect lines [13] ? or is this hysteresis extrinsic (due to the crystal inhomogeneities, dislocations, etc.). Somewhat like the hysteresis described by Néel [14] ? We feel that those questions, which are quite basic in the understanding of three dimensional modulated structures, and in particular in the problem of the piecewise analyticity of the thermodynamic functions [12] may be attacked from the point of view of their influence on the memory effect. Experimental as well as theoretical work is in progress on these questions and will be reported elsewhere.

Finally, we would like to suggest that a periodic defect concentration is expected to occur in practically all modulated structures, with a wide variation in relaxation times, orders of magnitude, etc., depending on the specific nature of each system, on the nature of the mobile defects, on the temperature at which modulated structures order, etc.. $\mathrm{NbSe}_{3}$ is a particularly interesting system in that respect [18]. The non linear response to applied electric field below the tempe- 
rature for CDW formation, as well as the periodic noise spectrum [18] can be understood provided one assumes a pinning potential of the CDW to the impurities. A random distribution of fixed impurities cannot provide the sinusoidal potential $\sim \sin \Phi$, where $\Phi$ is the CDW phase [19]. On the contrary, if a sufficient amount of impurities (or defects, or else, etc.) is able to relax in the periodic field of the modulation, as happens in thiourea, the appropriate $\sin \Phi$ potential arises most naturally from commensurability between CDW and impurity modulation, and accounts for the essential experimental features. In order to check this explanation, one should measure the threshold fields after quenching, annealing and look for time dependent effects linked with defect diffusion.

After this paper was completed, we received a preprint on the phase diagram of $\mathrm{NaNO}_{2}$ measured by neutron diffraction under electric field [20]. In this parent system of thiourea, the measured iso- $q$ lines are strikingly similar to those shown in figure 2 . As pointed out in reference [20], the iso- $q$ line curvature is well accounted for by introducing a term $\eta P_{x}^{2}(r)\left(\frac{\partial P_{x}(r)}{\partial z}\right)^{2}$ in equation (I.2), with $\eta>0$. We consider the results of reference [20] as a confirmation of the memory effect described here.

\section{Acknowledgments.}

We are pleased to thank Prof. V. Janovec, G. Montambaux and M. Barreto for stimulating discussions and F. Denoyer and D. Durand for showing us their neutron data prior to publication. J. Ferré is acknowledged for continuous interest while this work was completed. We thank Seb Doniach for helpful remarks.

\section{Appendix I.}

In the presence of the modulated impurity concentration the free energy of the modulated phase of thiourea is [10] :

with

$$
F=\int F_{\text {mod }} \mathrm{d} r+\int F_{\text {imp }} \mathrm{d} r
$$

$$
\begin{gathered}
F_{\text {mod }}=\frac{1}{2} A_{0} P_{x}^{2}(r)+\frac{1}{4} B P_{x}^{4}(r)+\frac{1}{2} \alpha\left(\frac{\partial P_{x}(r)}{\partial z}\right)^{2}+\frac{1}{4} \gamma\left(\frac{\partial^{2} P_{x}(r)}{\partial z^{2}}\right)^{2}-E_{x} P_{x}(r), \\
F_{\text {imp }}=C_{0} V_{0} b_{0} \sin 2 q_{0} r P_{x}^{2}(r) .
\end{gathered}
$$

In fact, (I.2) is a simplified version of the free energy for thiourea; in order to describe the modulated part of the phase diagram in the high field region, one needs to take into account a sixth order term [12] $\sim D P_{x}^{6}(r)$. Taking $D=0$, as we do here, allows a clearer derivation of the memory effect, and is only acceptable in thiourea at low fields $(E \lesssim 1 \mathrm{kV} / \mathrm{mm})$.

In(I. 2), $P_{x}(r)$ is the uniaxial polarization and $E_{x}$ the applied d.c. electrical field; $A_{0}=\left(T-T_{0}\right) / \Gamma$, $B$ and $\gamma$ are positive constants and $\alpha(T, E)$ is negative [15]. We consider that the phases of the system are described by a uniform polarization $P_{0}$ and a sinusoidal polarization of wavevector $q$. The Umklapp terms have been neglected. Then :

$$
\int F_{\text {mod }} \mathrm{d} r=\frac{1}{2} A_{0} P_{0}^{2}+A_{q}\left|P_{q}\right|^{2}+\frac{B}{4} P_{0}^{4}+3 B P_{0}^{2}\left|P_{q}\right|^{2}+\frac{3}{2} B P_{q}^{4}-E P_{0},
$$

where

$$
A_{q}=A_{0}-\alpha^{2} / 2 \gamma, \text { with } q_{0}^{2}=-\alpha / \gamma
$$

and

$$
\int F_{\text {imp }} \mathrm{d} r=b_{0} V_{0} C_{0}\left[\left|P_{q}\right|^{2} \delta\left(q-q_{0}\right)+P_{0}\left|P_{q}\right| \delta\left(q-2 q_{0}\right)\right] \text {. }
$$


We shall neglect the second term in (I.5) for two reasons : first of all, in most of the modulated phase $P_{0}^{2} \ll\left|P_{q}\right| P_{0}$, and secondly, there is no way to mark the system at $q_{0}$, and test an echo at $2 q_{0}$ because $\frac{2 \pi}{9 b}<q(T)<\frac{2 \pi}{7 b}$.

Then $P_{0}$ and $P_{q}$ are determined by $\partial F / \partial P_{0}=0$ and $\partial F / \partial P_{q}=0$, i.e. :

$$
\left.\begin{array}{l}
A_{0} P_{0}+B P_{0}^{3}+6 B\left|P_{q}\right|^{2} P_{0}+E=0 \\
A_{q}+3 B P_{0}^{2}+3 B\left|P_{q}\right|^{2}+b_{0} V_{0} C_{0} \delta_{q, q_{0}^{1}}=0 .
\end{array}\right\}
$$

In (I.6), the terms of order $P_{0}^{3}$ are negligible in the modulated phase at low and intermediate field values $(E \lesssim 1 \mathrm{kV} / \mathrm{mm}$ in deuterated thiourea).

At last, we have :

$$
\left|P_{q}\right|^{2}=-\frac{A_{q}}{3 B}-P_{0}^{2}-\frac{b_{0} V_{0} C_{0}}{3 B} \delta_{q, q_{0}}
$$

and at low fields :

$$
P_{0}=E\left[A_{0}-2 A_{q}-2 b_{0} V_{0} C_{0} \delta_{q, q_{0}}\right]^{-1}
$$

in the limit $A_{0}-2 A_{q} \gg 2 b_{0} V_{0} C_{0}$ we obtain the result of the text. The latter is thus seen to be a weak coupling limit, as emphasized in the conclusion.

\section{References}

[1] Moudden, A. H., Denoyer, F., Lambert, M., Fitzgerald, W., Solid State Commun. 32 (1979) 933.

[2] Hamano, K., Hishinuna, T., Ema, K., J. Phys. Soc. Japan 50 (1981) 2666.

[3] Almairac, R., Ribet, M., Ribet, J. L., Bzouet, M., J. Physique Lett. 41 (1980) L-315.

Regis, M., Ribet, J. L., JAMet, J. P., J. Physique Lett. 43 (1982) L-333.

MARION, G., J. Physique 42 (1981) 469.

[4] SChneCK, J., Denoyer, F., Phys. Rev. B 23 (1981) 383.

"[5] Comes, R., Shirane, G., Shapiro, S. M., Garito, A. F. and Heeger, A. J., Phys. Rev. B 14 (1976) 2376.

[6] Hamano, K., Ema, K., Hirotsu, S., Ferroelectrics 36 (1981) 343.

[7] Macmillan, W. L., Phys. Rev. B 14 (1978) 1496.

Rice, T. M., Whitehouse, S., LittleWOod, P., Phys. Rev. B 24 (1981) 2751.

LeE, P. A. and RICE, T. M., Phys. Rev. B 19 (1979) 3970.

[8] Badoz, J., Billardon, M., Canit, J. C., Russel, M. F., J. Optics 8 (1977) 373.

[9] Jamet, J. P., Quittet; A. M., Moudden, A. H., Recent Developments in Condensed Matter Physics (Plenum Press, J. T. Devreese editor) 1981, Vol. 4, p. 61.

[10] a) JAMET, J. P., J. Physique Lett. 42 (1981) L-123.

b) JAMet, J. P., Lederer, P., Moudden, A. H., Phys. Rev. Lett. 48 (1982) 442.

[11] Denoyer, F., Moudden, A. H., Lambert, M., Ferroelectrics 24 (1980) 48.

[12] Barreto, M., LeDerer, P., Jamet, J. P., To be published.

[13] JANOVEC, V., Ferroelectrics 35 (1981) 105 and private communication.

[14] NéEL, L., J. Physique Radium 13 (1952) 249.

[15] Lederer, P. and Chaves, C. M., J. Physique Lett. 42 (1981) L-127.

[16] Moudden, A. H., Svensson, E. C. and Shirane, G., Phys. Rev. Lett. 49 (1982) 557.

[17] Denoyer, F., Durand, D., Currat, R., Vettier, C., To be published.

[18] Monceau, P., Richard, J. and Renard, M., Phys. Rev. B 25 (1982) 931 and references therein.

[19] EFetov, K. B. and Larkin, A. I., Sov. Phys. JETP 45 (1977) 1236.

[20] Durand, D., Denoyer, F., Lefur, D., Currat, R. and Bernard, L., to appear in J. Physique Lett. 44 (1983). 